\title{
Research on Recovery System of Distribution Network Fault
}

\author{
Xuedong Zhang ${ }^{1}$, Yating Zhao ${ }^{2}$, Ling $\mathrm{Liu}^{3}$, Kai Sun ${ }^{4}$ Ye $\mathrm{Ma}^{5}$ \\ ${ }^{1345}$ State Grid Shanxi Electric Power Company Jinzhong Power Supply \\ Company, 030600 \\ ${ }^{2}$ School of Electrical Automation Engineering, Nanjing Normal \\ University, Nanjing, China, 210042
}

\begin{abstract}
Distribution automation is the necessary means and shortcuts to improve distribution network power supply reliability, security and service quality, improve work efficiency, reduce operating costs. Based on the development of distribution automation, this paper develops the fault recovery system of distribution network, and studies the various problems involved in the fault recovery system.

Keywords: recovery system, distribution network, fault
\end{abstract}

\section{Introduction}

Fault detection, isolation and recovery system (referred to as fault recovery system, abbreviated as FDIR) is an important application function of distribution management system (DMS). The main function is based on the information of switch tripping and fault indication Information and intelligent software to analyze and judge the fault occurred in the feeder of the distribution network in real time and put forward the correct and effective strategy to help the dispatchers to accurately determine the fault location, isolate the fault area and restore the power supply in the non-fault area as far as possible. Fault losses are minimized. The main feature is the ability to consider the number of switching operations, feeder margin, load recovery, network constraints, user priorities and other factors, the proposed optimal power supply recovery program. 
It is a multi-objective, multi-time, multi-combinatorial and multi-constrained nonlinear optimization problem. The final solution is a series of switch action combinations. However, the complexity of the problem determines that it can not be perfectly solved mathematically. At present, the main idea of solving this problem is the application of artificial intelligence and numerical calculation of the combination of technology, typical methods are: the use of heuristic search method to find possible recovery program, and numerical calculation to determine the viable or preferred recovery program; the use of fuzzy mathematical principles Fuzzy programming or fuzzy evaluation, to determine the optimal solution; the use of expert system technology, build rules and reasoning; use of genetic algorithms, the establishment of evaluation function, seeking the evaluation function under the optimal solution.

\section{The Fault Recovery Algorithm}

Fault recovery of distribution network is an extremely complex real-time decision-making process, involving a large number of uncertainties and the existing online numerical analysis technology is difficult to provide effective help, mainly by the dispatcher based on field experience to make decisions. When the system fails, a variety of information flocked to a short time, this time need to control personnel as much as possible to control the situation, to avoid the expansion of the accident, and restore as much load in a short time, at this time, dispatcher Not only have a solid professional knowledge and rich operational experience, familiar with a variety of operating modes, operating procedures and safety parameters of the limit, but also to maintain a clear head. In fact, due to the lack of practical experience in dealing with the accident, coupled with the heavy burden of heavy responsibilities, the face of such complex and urgent scenes, requiring dispatchers to experience and intuition only take correct and effective measures, and minimize the loss of the accident is very difficult.

\subsection{Heuristic search}

Heuristic search is a commonly used method of failover. Heuristic search is a heuristic rule-guided search model, relying on specialized information to simplify the search and improve efficiency. At the same time, the evaluation function is used to evaluate the search space and realize the priority search. Most of the heuristic search failure recovery methods are based on the operation of the switch, using heuristic rules to define the special operating standards, test a large number of candidate solutions. Heuristic search is widely used, usually the best combination of algorithms and rules, using the minimum switching operation for the optimal solution, thereby reducing operating time. Heuristic rules are out-ofthe-box rules that are different from those of other artificial intelligence methods, and are not constrained by the structure of the system. However, heuristic rules based on field experience and technical analysis greatly determine the efficiency of the algorithm, and cannot guarantee the optimal solution every time. 


\subsection{The expert system}

Expert System (Expert System) is the field of artificial intelligence, the most mature of a discipline, became an international hot spot. The advantage of the expert system is that the problem of power system fault recovery needs to be solved by the experience and heuristic rules of the dispatcher, which is difficult to be expressed by the mathematical model, which is the advantage of the expert system. The expert system also has some shortcomings. It is very difficult to establish a complete knowledge base. The knowledge base is often too large. At the same time, the learning ability is weak, and the ability of system response and fault tolerance is poor. How to further improve the performance of expert system has been the attention of scholars at home and abroad, the control center using expert systems to achieve fault recovery strategy, and sensitivity analysis method of self-test system constraints, limiting the size of the search space and improve search efficiency.

\subsection{Integer programming method}

Integer programming method is also often used to achieve power recovery, it will be the problem to be solved in the standard form of mathematical expression of the objective function and inequality constraints for mathematical solution. The advantage of this algorithm is that when the objective function of the optimal solution, it will be able to find. But with the power to expand the region, this algorithm will greatly extend the time.

\section{The Fault Recovery Model of Distribution Grid}

Distribution network fault recovery can be roughly divided into three kinds of recovery model, the following separately discussed.

\subsection{Early failure recovery mode}

In the early stages of low automation, fault recovery relies on fault indicators installed on the distribution lines. After the failure, the staff relies on the fault indicator to find the fault location, the use of column switching equipment manually isolate the fault area, manual recovery of non-fault area power supply.

\subsection{Distribution automation fault recovery model}

This mode (mainly referred to as DA mode) mainly depends on the hardware devices installed in the distribution network, through the switching function and protection time, Diagnosis, isolation and recovery. This recovery model is characterized by the early distribution network planning, distribution network structure and the degree of automation of distribution equipment, but generally only applies to a simple junction network, and cannot consider the actual load level and network operating constraints. 


\subsection{The failure recovery mode of power distribution management system}

This mode (referred to as DMS mode) is mainly used in the distribution control center fault recovery software to achieve fault detection, isolation and power supply recovery. When the fault occurs in the distribution network, the fault recovery software of the DMS performs logical reasoning based on the fault information collected by the FTU and transmitted to the SCADA real-time database of the power distribution control center through the communication system to determine the fault location and determine the fault isolation and restoration of power supply Operation steps, and then submitted to the operation sequence in the form of SCADA system, manual or automatic implementation. This recovery model is applicable to any distribution network, which can deal with some special situations (such as multiple faults), can consider the actual load level and network constraints, but with the equipment reliability and software functions are closely related.

\section{The Database Design of the Fault Recovery System}

\subsection{The database design}

The core of the fault recovery is the rapid formation and evaluation of the restoration scheme, and the formation of the restoration scheme is based on the network connectivity analysis. Therefore, in order to carry out effective reasoning, we must first establish a network structure database suitable for inference.

On the other hand, the power system elements can be classified into a switching element (logic element) and a non-switching element type in terms of the communication property of the element. The state of the switching element (closing, breaking) determines the connectivity between the two ends of the switch, thus determining the connectivity between the non-switching elements and, ultimately, the connectivity of the network. In principle, any form of connectivity analysis can be performed as long as it is based on the association table between nodes and components.

However, in the process of distribution network fault recovery, if the search table is directly guided by the most primitive node-element association table provided by the network database, there are many types of distribution network elements, and the number is large, It will affect the search efficiency and search speed, and it is difficult to establish the corresponding reasoning model and control strategy.

In the distribution network, a distribution feeder is generally divided into several sections by feeder section switches, each section also contains a number of branch line segment (SEC) and user load (LD). Feeder contains a large number of components, but contains a small number of sections, usually 2 to 4 . During the fault recovery process, the segment is the smallest controllable part of the feeder, the internal connection relationship is unchanged, can be considered as a whole. Therefore, when performing network connectivity analysis, a segment can 
be treated as a component with multiple end nodes, regardless of its internal component composition and connection relationships. When detailed analysis calculations are required, for example, when power flow calculations are required to verify the candidate recovery scheme, then consider the specific section within the knot. In this way, by introducing the concept of the section, the physical model of the distribution network can be simplified, and the reasoning speed and efficiency of fault recovery can be improved.

\subsection{The database design structure}

Based on the above design idea, the distribution network is regarded as composed of two types of components: switching elements and sector components. Segment elements are composed of their containing elements, which are the physical elements of the original network database, namely single-ended, double-ended, and three-terminal elements. Through the introduction of the concept of the segment, the association table between the original node and the component in the network database is decomposed into the relation table of node and section, section and element. When performing network analysis (such as live analysis, route search), the use of nodes and sections of the association table to perform the search; in the need for detailed network calculation and analysis, and further use of the association table with the formation of computing models .

\section{Conclusion}

With the development of the national economy, the power system is becoming more and more complex, and the system failure caused by various accidental factors is inevitable. At the same time, the dependence of the whole society on electric energy is increasing. The reliability and power quality requirements are getting higher and higher. Therefore, it is necessary to develop a more complete and practical fault recovery system for HV distribution network.

\section{References}

[1] Tan Dundu, Tan Xiaotian. Power Distribution Management System DMS Hunan Electric Power, 55(12), pp.68-70, 2011

[2] Chen Jingcheng, $\mathrm{Xu}$ Dechao, Yu Erkeng. Distribution Network Fault Recovery System Power System Automation, 8 (5), pp.87- 91, 20013

[3] Lin Gongping. Technical Solution of Distribution Feeder Automation Solution Power System Automation, 12(10), pp. 18- 20, 2012

[4] Sun Fujie, Wang Gangjun, Li Jianglin. Comparison and Optimization of Feeder Automation Fault Handling Mode for Distribution Networks 9(6), pp.5861,2011

[5] Sun Fujie, He Junjia, Zou Jiyan. Research and application of power supply technology based on recloser and segmentation for $10 \mathrm{kV}$ ring network, 8(4), pp.85-87, 2014 